\title{
A Case Report of Still's Disease in the Adult
}

\author{
Leila A. Cardoso ${ }^{\text {a, b }}$, Fatima Silva ${ }^{\text {a }}$, Joana Paixao ${ }^{\text {a }}$, \\ Adriano Rodrigues ${ }^{\mathrm{a}}$
}

\begin{abstract}
Adult Still's disease (ASD) is a rare systemic inflammatory disorder of unknown etiology, typically characterized by a clinical triad (daily spiking high fevers, evanescent rash, and arthritis) and a biological triad (hyperferritinemia, hyperleucocytosis with neutrophilia and abnormal liver function test). There are no specific diagnostic tests for ASD, so the diagnosis of ASD remains one of exclusion and the differential diagnosis may be lengthy. We present a case of an adultonset Still's disease with previous admissions for fever of unknown origin with a 2-month history of fever and systemic symptoms. The patient posteriorly presented polyarthralgies and cutaneous rash. The study disclosed anemia, inflammatory markers and hepatic enzymes elevation, and negative serological and immunological studies. The introduction of corticotherapy resolved symptoms and laboratories alterations. Adult-onset Still disease is a heterogeneous and rare disease and the lack of serologic markers as a true gold standard makes diagnosis difficult.
\end{abstract}

Keywords: Adult Still's disease; Fever; Rash; Hyperferritinemia; Polyarthralgies

\section{Introduction}

Adult Still's disease (ASD) is a systemic inflammatory disorder of unknown etiology, typically characterized by a clinical triad (daily spiking high fevers, evanescent rash, and arthritis) and a biological triad (hyperferritinemia, hyperleucocytosis with neutrophilia and abnormal liver function test) [1].

ASD is a rare disorder, known to exist worldwide, with equal distribution between the sexes, and with three quarters of patients reporting disease onset between 16 and 35 years of age [2].

The laboratory findings in ASD reflect the systemic inflammation and cytokine cascade present, and none of the find-

Manuscript submitted May 12, 2017, accepted May 23, 2017

aServico de Medicina Interna B, Centro Hospitalar Universitario de Coimbra, Coimbra, Portugal

${ }^{b}$ Corresponding Author: Leila Amaro Cardoso, Servico de Medicina Interna B, Hospital Geral do Centro Hospitalar Universitario de Coimbra, 3046-853 Coimbra, Portugal. Email: leilaamarocardoso@gmail.com

doi: https://doi.org/10.14740/jmc2847w ings are specific for ASD. There is no association with rheumatoid factor or antinuclear antibody positivity [3].

There are no specific diagnostic tests for ASD. The diagnosis of ASD remains one of exclusion and the differential diagnosis may be lengthy. Infectious, neoplastic, autoimmune diseases or drug hypersensitivity reactions can mimic the clinical manifestation of ASD. Therefore, several sets of different classification criteria have been proposed for ASD $[3,4]$. The classification criteria proposed by Yamaguchi et al published in 1992 are the most widely used [1].

Treatment options include non-steroid anti-inflammatory drugs (NSAIDs) and aspirin, glucocorticoids, and immunomodulating drugs. Most patients require steroids at some point in the course of their ASD; the usual prednisone dose is $0.5-1.0 \mathrm{mg} / \mathrm{kg} / \mathrm{day}$. Responses to steroid therapy range from $76 \%$ to $95 \%[2,5]$.

\section{Case Report}

We present a case of a 26-year-old female patient, admitted in our outpatient hospital with a constitutional syndrome, with fever, asthenia and polyarthralgia 2 months long. At the moment of the first appointment, arthritis of small joints, axillary and cervical adenopathies, with anemia, thrombocytosis, neutrophilia and elevation of inflammatory markers (C-reactive protein and erythrocyte sedimentation rate) and transaminases were noted. The patient was already on steroid therapy (prednisolone $20 \mathrm{mg}$ per day) since the clinical outbreak. A CT scan was therefore realized to further evaluation, having confirmed axillary, cervical and mediastinal adenopathies with $10-30$ $\mathrm{mm}$; no hepato- or splenomegaly was documented. Hence, a biopsy to one for the cervical lymph nodes was preformed, yet with no certain conclusions, since the histologic features were compatible with Hodgkin's lymphoma, but in terms of immunophenotyping characteristics, it was in favor of a large B-cell lymphoma. The patient was then hospitalized to better understand the clinical features. During the time of admission, patient had high fever with no patterns, unresponsive to therapeutics, associated with maculopapular evanescent rash. An anatomopathological review of the first histological sample was requested, having shown a reactive lymphadenitis. The possibility of an infectious disease was studied and excluded; the autoimmune laboratorial workout was also negative.

At the moment, we have a patient with a high fever, associated with polyarthritis, evanescent rash and reactive lymphadenitis. Infectious and lymphoproliferative conditions were excluded. The laboratorial workout showed inflammatory 
Table 1. Yamaguchi Criteria - Five or More Criteria With at Least Two Major Positive

\begin{tabular}{ll}
\hline Major criteria & Minor criteria \\
\hline Fever $\left(>39^{\circ} \mathrm{C}\right)$ & Pharyngitis \\
Arthralgia (over 2 weeks long) & Lymphadenopathy/splenomegaly \\
Cutaneous rash & Hepatic dysfunction \\
\hline Leucocytosis with neutrophilia & ANAs and RF negative \\
\hline
\end{tabular}

markers and transaminases elevation. Therefore, the possibility of an ASD was accessed. Further laboratorial study showed hyperferritinemia of 5,339.9 $\mu \mathrm{g} / \mathrm{dL}$. The patient scored on Yamaguchi criteria, having the ASD diagnosed. Prednisolone $0.5 \mathrm{mg} / \mathrm{kg} /$ day was then started, and great response to steroid therapy was shown, with total resolution of the clinical manifestations.

\section{Discussion}

Despite the improvement in diagnostic techniques, the undetermined febrile syndrome (UFS) remains a challenge to overcome, comprising $50 \%$ of the cases without clear etiology [6]. ASD represents one of the possible diagnoses among UFS, only accessible excluding a long list of other possibilities. Hence, until today, ASD remains a difficult diagnosis, relying in six different sets of classification criteria (Glodman, Calabro, Cush, Reginato, Kahn and Yamaguchi) [7]. The Yamaguchi criteria (Table 1) are the ones recognized for their superior accuracy, as it was shown in Masson et al study in 1996 [7]. The two greatest obstacles concerning these criteria are: the fact that, despite being a diagnosis of exclusion, there is not a clear set of diagnosis to exclude before assuming ASD, nor helpful complementary diagnosis techniques to help supporting the hypothesis; the other limitation is the absence of ferritin levels (or its glycosylated form) in the criteria [8].

Therefore, the frequent delay on the diagnosis brings no surprises and our case is no exception. The lack of specificity of the symptoms and signs, and their absence of synchronicity concerning the cutaneous manifestations, represent a strong contributor to the difficulties on this diagnosis [9-11].

In our case, after excluding other causes of febrile syndrome, we recognized six aspects of Yamaguchi criteria: high fever with more than 3 weeks of evolution, leucocytosis with neutrophilia associated with atypical cutaneous rash (major criteria); splenomegaly, transaminases and rheumatoid factor elevation and negativity antinuclear antibodies (minor criteria). We have to enlighten the long period of clinical evolution (5 years) without compromising the patient's general wellbeing [12].

The search for a sensible/specific biochemical marker for this disease is still a long awaited discovery. Consistent data towards the predominant role of macrophage activity in Still's pathophysiology have been found, since high levels of ferritin, IL-6, -8, -18 and the TNF-alpha are frequently recognized [13].

The treatment generally relies on NSAIDs and steroids, requiring no further more potent immunosuppressors. In some refractory cases, hydroxychloroquine, gold salts, methotrexate and cyclosporine have been used with limited data $[14,15]$.

In our case, with a mild articular damage (known for being the major indicator of a poorer prognosis), low-dose corticoid and NSAIDs were used with resolution of all symptoms. During a 2-year follow-up, no recurrences were found.

\section{Conclusion}

We present this case of ASD to enlighten the difficulties concerning the diagnosis and the need of more accurate classification criteria. The lack of high sensibility methods to recognize ASD delays the diagnosis, therefore comprising the correct treatment to improve the outcome. The intricacy of the symptoms and the lack of accurate diagnostic techniques make the clinical approach to ASD patients the hallmark to the right management of this condition.

\section{Acknowledgments}

Joao Pedro Abreu Hospital Santa Maria Maior, Barcelos, Portugal.

\section{References}

1. Bywaters EG. Still's disease in the adult. Ann Rheum Dis. 1971;30(2):121-133.

2. Efthimiou P, Paik PK, Bielory L. Diagnosis and management of adult onset Still's disease. Ann Rheum Dis. 2006;65(5):564-572.

3. Yamaguchi M, Ohta A, Tsunematsu T, Kasukawa R, Mizushima Y, Kashiwagi H, Kashiwazaki S, et al. Preliminary criteria for classification of adult Still's disease. J Rheumatol. 1992;19(3):424-430.

4. Masson C, Le Loet X, Liote F, Dubost JJ, Boissier MC, Perroux-Goumy L, Bregeon C, et al. Comparative study of 6 types of criteria in adult Still's disease. J Rheumatol. 1996;23(3):495-497.

5. Wouters JM, van de Putte LB. Adult-onset Still's disease; clinical and laboratory features, treatment and progress of 45 cases. Q J Med. 1986;61(235):1055-1065.

6. Bleeker-Rovers CP, Vos FJ, de Kleijn EM, Mudde AH, Dofferhoff TS, Richter C, Smilde TJ, et al. A prospective multicenter study on fever of unknown origin: the yield of a structured diagnostic protocol. Medicine (Baltimore). 2007;86(1):26-38.

7. Crispin JC, Martinez-Banos D, Alcocer-Varela J. Adultonset Still disease as the cause of fever of unknown origin. Medicine (Baltimore). 2005;84(6):331-337.

8. Fautrel B, Zing E, Golmard JL, Le Moel G, Bissery A, Rioux C, Rozenberg $S$, et al. Proposal for a new set of classification criteria for adult-onset still disease. Medicine (Baltimore). 2002;81(3):194-200.

9. Affleck AG, Littlewood SM. Adult-onset Still's disease with atypical cutaneous features. J Eur Acad Dermatol Venereol. 2005;19(3):360-363. 
10. Tomaru K, Nagai Y, Ohyama N, Hasegawa M, Endo Y, Tamura A, Ishikawa O. Adult-onset Still's disease with prurigo pigmentosa-like skin eruption. J Dermatol. 2006;33(1):55-58.

11. Wolgamot G, Yoo J, Hurst S, Gardner G, Olerud J, Argenyi Z. Unique histopathologic findings in a patient with adultonset Still disease. Am J Dermatopathol. 2007;29(2): 194-196.

12. Cush JJ, Medsger TA, Jr., Christy WC, Herbert DC, Cooperstein LA. Adult-onset Still's disease. Clinical course and outcome. Arthritis Rheum. 1987;30(2):186-
194.

13. Arlet JB, Le Thi Huong DB, Pouchot J, Piette JC. [Current concepts on the physiopathology of adult-onset Still's disease]. Rev Med Interne. 2005;26(7):549-556.

14. Singh S, Samant R, Joshi VR. Adult onset Still's disease: a study of 14 cases. Clin Rheumatol. 2008;27(1):35-39.

15. Omagari $\mathrm{K}$, Matsunaga $\mathrm{Y}$, Yamashita $\mathrm{H}$, Nishiyama $\mathrm{H}$, Hazama $\mathrm{H}$, Oda $\mathrm{H}$, Isomoto $\mathrm{H}$, et al. Successful treatment with cyclosporin in adult-onset Still disease manifesting as acute hepatitis with marked hyperferritinemia. Am J Med Sci. 2003;326(3):148-151. 